\title{
Closure modeling and direct simulation of vegetation drag in flow through emergent vegetation
}

\author{
Su Jin $\mathrm{Kim}^{1}$ and Thorsten Stoesser ${ }^{1}$ \\ Received 17 February 2011; revised 26 August 2011; accepted 1 September 2011; published 13 October 2011.
}

[1] This paper presents numerical simulations of flow through emergent vegetation. Two simulation strategies are evaluated, (1) Reynolds-averaged Navier-Stokes (RANS)- based simulations employing a vegetation closure model and (2) low-resolution large-eddy simulation (LES). RANS-based models offer efficiency in terms of computational resources, however, it is demonstrated herein that the accuracy of RANS models depends strongly on empirical parameters of the corresponding vegetation closure model. The method of low-resolution LES is an efficient alternative to a fully resolved LES, simulates vegetation drag directly, and does not require empirical parameter input. Predictions of the vegetative flow resistance of emergent vegetation using low-resolution LES are in fairly good agreement with measurements, in particular for low and moderate vegetation densities. This is because prevailing stream- and spanwise-velocity gradients, vertical velocity profiles, and recirculation zones are calculated with reasonable accuracy.

Citation: Kim, S. J., and T. Stoesser (2011), Closure modeling and direct simulation of vegetation drag in flow through emergent vegetation, Water Resour. Res., 47, W10511, doi:10.1029/2011WR010561.

\section{Introduction}

[2] Vegetation on the banks or floodplains of rivers and streams significantly affects the horizontal and vertical velocity distributions as well as the turbulence statistics. Accurate quantification of the bulk effects of flow-vegetation interaction is a significant challenge in the field of openchannel hydraulics and is, for instance, of great importance for the design of flood protection or stream restoration schemes [Stoesser et al., 2003].

[3] Over the last four decades, the tool of computational fluid dynamics (CFD) has been developed and refined. CFD models are able to provide accurate flow predictions of many flows of practical hydraulic and/or hydrological interest. In general, the methods of direct numerical simulation (DNS), large-eddy simulation (LES), and Reynolds-averaged-Navier Stokes (RANS) have evolved. LES lies between the extreme approaches of DNS, where all turbulent fluctuations are computed and no turbulence model is required, and steady RANS, where only the mean velocity field is computed and all the unsteady effects of turbulence are accounted for by a turbulence model. Nowadays, RANS is considered a computationally efficient engineering tool, while DNS and LES are much more expensive and are mainly used in research. LES and DNS offer a substantial increase in accuracy over timeaveraged approaches, particularly when large-scale turbulent structures dominate the flow [e.g., Rogallo and Moin, 1984].

[4] In the mid 90s, various RANS models were developed to simulate the flow through vegetation and different turbulence models were employed to calculate the Reynolds

\footnotetext{
${ }^{1}$ School of Civil and Environmental Engineering, Georgia Institute of Technology, Atlanta, Georgia, USA.
}

Copyright 2011 by the American Geophysical Union. 0043-1397/11/2011WR010561 stresses that are a result of Reynolds averaging. RANS models are operated on coarser grids and the additional form drag due to vegetation is accounted for through subgrid forces that are added to the momentum and turbulence model transport equations. This treatment should be referred to as vegetation closure model; RANS models offer reasonable accuracy in the prediction of the timeaveraged flow field [Choi and Kang, 2004; Defina and Bixio, 2005; Fischer-Antze et al., 2001; Lopez and Garcia, 2001; Naot et al., 1996; Neary, 2000, 2003; Nicholas and McLelland, 2004; Tsujimoto and Shimizu, 1994], but agreement with measured turbulence quantities is sometimes poor [Defina and Bixio, 2005; Neary, 2003]. This is mainly because steady RANS models do not resolve flowvegetation interaction. Vortex shedding and local velocity gradients are absent, hence RANS models require additional drag-related terms in the turbulence models' transport equations to account for vegetation-related turbulence production and its dissipation. Drag force terms in the momentum and drag-related terms in the turbulence model transport equations, require a priori estimates of the drag coefficient and additional empirical constants.

[5] Most vegetation closure models use the drag force formula, i.e., $F_{D}=0.5 \rho u_{0}^{2} A_{P} C_{D}$, with $\rho$ being the density of the working fluid, $u_{0}$ the free stream velocity, $A_{P}$ the projected area of the plant, and $C_{D}$ the drag coefficient, which is an empirical parameter. In many experimental investigations of flow through vegetation the cylinder analogy is made use of, i.e., vegetation can be idealized as a bunch of rigid circular cylinders. The calculation of vegetation drag is then straightforward and the only uncertainty is in the selection of $C_{D}$. However, even though many RANS based studies that used the drag force approach report a reasonably good match of predicted velocity profiles with observed ones, some inaccuracies were found in the prediction of the 
head loss in the system. The main reason is that RANS models have adopted a "universal" drag coefficient, $C_{D}$, without consideration of its variation as a function of vegetation density and stem Reynolds number [Defina and Bixio, 2005]. For instance, Fischer-Antze et al. [2001] modeled emergent floodplain vegetation in a compound channel and because of the lack of knowledge of $C_{D}$ they assumed the drag coefficient to be $C_{D}=1.0$, reasoning that it is a valid value over a wide range of cylinder (stem) Reynolds numbers, $R e_{D}$ for flow around an isolated cylinder. However, the drag coefficient in an array of cylinders may differ from the one of an isolated cylinder. In a recent laboratory study, Tanino and Nepf [2008] demonstrated that the drag coefficient is a function of both vegetation density and stem Reynolds number, and that it can deviate largely from unity.

[6] One way of avoiding the $C_{D}$ empiricism in numerical models is to resolve the vegetation explicitly through the numerical grid. This was recently done by Stoesser et al. [2009, 2010], who performed several LES of the flow through submerged and emergent vegetation (idealized by rigid cylinders). They imposed the no-slip boundary condition on all solid boundaries, including the surface of the cylinders. In that way, flow-vegetation interaction was explicitly calculated. The simulations were validated with laboratory data and fairly good agreement was found. After successful validation, Stoesser et al. [2010] quantified the drag force of each cylinder. They confirmed the findings of Tanino and Nepf [2008], i.e., $C_{D}$ depends on both stem Reynolds number and vegetation density, and provided evidence that this trend is valid at stem Reynolds numbers greater than those examined by Tanino and Nepf [2008].

[7] A summary of numerical simulations of flow through vegetation is given in Table 1, which includes the mention of the respective vegetation closure model and the authors' choice of the closure coefficient/parameters. Table $1 \mathrm{dem}-$ onstrates that almost all numerical models to date employ the drag-force concept, which requires a priori knowledge of the drag coefficient. $C_{D}$ was selected either on the basis of experiments [e.g., Lopez and Garcia, 2001], or it was calibrated to match experimental data [e.g., Wu et al., 2005], or it was set to a fixed value, e.g., $C_{D}=1.0$ by Fischer-Antze et al. [2001] because of a lack of knowledge of the real value. The LES by Stoesser et al. [2009, 2010] do not employ a vegetation closure model but the vegetation is idealized as rigid cylinders and such a treatment is computationally very demanding. Hence, LES is limited to relatively simple (vegetation) geometries and relatively low stem Reynolds numbers because of its need for very fine grids.

[8] The first objective of this study is to assess the importance of a priori knowledge of the empirical drag coefficient in flow through emergent vegetation (here idealized as cylinder matrices) when using RANS with a vegetation closure model. The second objective is to evaluate a lowresolution LES strategy that is able to simulate vegetation drag directly. Low-resolution LES does not rely on empirical parameters and is computationally cheaper than a fully resolved LES. Simulations are carried out for different numerical and physical experiments of uniform flow through emergent vegetation at varying vegetation density and stem Reynolds numbers.

\section{Numerical Framework}

[9] The two numerical strategies to predict the hydrodynamics of turbulent flow through emergent vegetation evaluated in this study are (1) a RANS-based approach with a subgrid vegetation closure model and (2) a novel lowresolution large-eddy simulation (LES) approach.

\subsection{Reynolds-Averaged Navier-Stokes-Based Approach}

[10] The RANS model, validated by Fischer-Antze et al. [2001], is employed to calculate the time-averaged flow through emergent vegetation, which, in this study, is emulated by a matrix of cylinders. The program solves the RANS equations with the finite-volume approach on a structured, nonorthogonal grid. A second-order upwind scheme approximates the convective terms in the momentum equations, whereas diffusive terms are approximated with a central differencing scheme. The SIMPLE method [e.g., Patankar, 1980] couples the pressure to the velocity field and the standard $k-\varepsilon$ turbulence closure model evades the explicit calculation of the Reynolds stresses appearing in the RANS formulation of the Navier-Stokes equations. A force term is added to the right-hand side of the momentum equations to account for the momentum loss due to vegetation. The implementation is described in detail by Fischer-Antze et al. [2001]. While the channel bed is treated with a no-slip condition, at the spanwise boundaries and the rigid-lid, free surface boundary a slip condition is applied. Periodic boundary conditions are used in the streamwise direction to induce a fully developed uniform flow.

\subsection{Low-Resolution Large-Eddy Simulation Approach}

[11] The low-resolution LES is developed as an alternative to high-resolution LES to simulate flow through emergent vegetation but at lower computational cost. The low-resolution LES is based on the LES code Hydro3D [Stoesser and Nikora, 2008]. The code solves the filtered Navier-Stokes equations with the finite volume method for incompressible fluid flow on Cartesian grids. Convective and diffusive fluxes are approximated with central differences of secondorder accuracy, and time advancement is achieved by a third order, three step, explicit Runge-Kutta scheme. The Poisson equation for coupling the pressure to the velocity field is solved iteratively with the SIP method of Stone [1968] after the third Runge-Kutta step to ensure a divergence-free flow field. The subgrid-scale stresses appearing in the filtered Navier-Stokes equations are computed using the dynamic version [Germano et al., 1991] of the original Smagorinsky model [Smagorinsky, 1963]. The wall boundary is treated with a no-slip condition using wall functions and the water surface is fixed as a rigid lid, and a slip condition is applied there. Periodic conditions are used at the stream- and spanwise boundaries. To treat the vegetation, the low-resolution LES code uses a simplified immersed boundary method on a Cartesian grid, similar to the one introduced by Stoesser [2010]. This is in contrast to the high-resolution LES in which each vegetative element is resolved explicitly with a body-fitted, curvilinear grid. The grids of both RANS and low-resolution LES are depicted in Figure 1. 


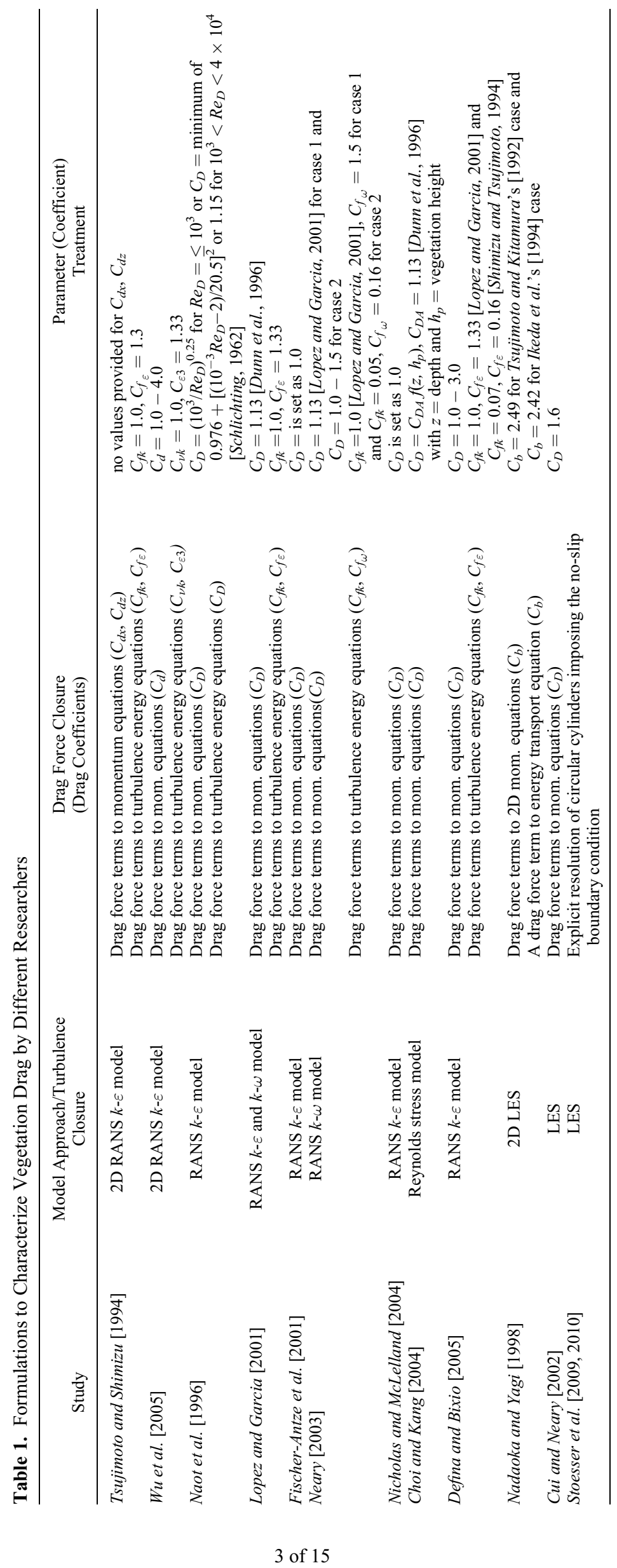


(a)

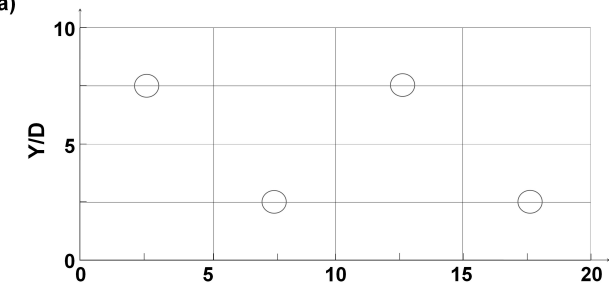

(b)

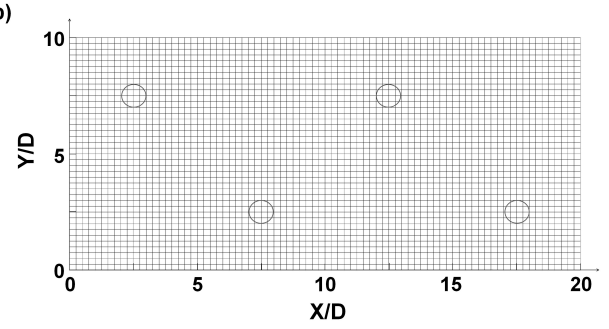

Figure 1. Coarsest numerical grid of the (a) RANS simulations and (b) low-resolution LES for the $\phi=0.016$ case.

[12] Figure 2 illustrates the concept of the novel immersed boundary method applied herein to account for the rigid cylinders, which are immersed in the low-resolution LES grid. In a preprocessing step, three types of computational cells around a circular obstacle are identified in the Cartesian grid, i.e., fluid cells, cut cells, and inside cells. The fluid volume fraction, $V_{f}$, which is defined as the ratio of the volume of fluid to the total volume of a cell, is computed for each cell. Fluid cells with $V_{f}=1.0$ do not require any treatment, while inside cells (i.e., $V_{f}=0.0$ ) are blocked out of the computation so that $u_{i}=0.0$. The velocity in the cut cells, $u_{i}$, is updated after the third and final Runge-Kutta step by multiplying the value from the previous RungeKutta step with the volume fraction $V_{f}$. Before moving to the next time step, a divergence-free flow field is ensured by the solution of a Poisson equation for the pressure followed by a final update of the velocity field in the fluid cells. The proposed immersed boundary method should be considered first-order accurate but is stable and oscillation free, because the gradients at the fluid-cylinder interface are somewhat smeared out. The use of a Cartesian grid together with the immersed boundary method is advantageous in two ways. First, solvers for Cartesian grids are more efficient than for body-fitted, curvilinear grids. Second, the immersed boundary method allows for very complex vegetation arrangements, e.g., a random distribution of vegetation stems, in which stems can be placed very close to each other. For such scenarios, body-fitted grids are inappropriate or even impossible.

\section{Test Cases}

[13] In order to test and validate the RANS and lowresolution LES approaches, data of the laboratory experiments

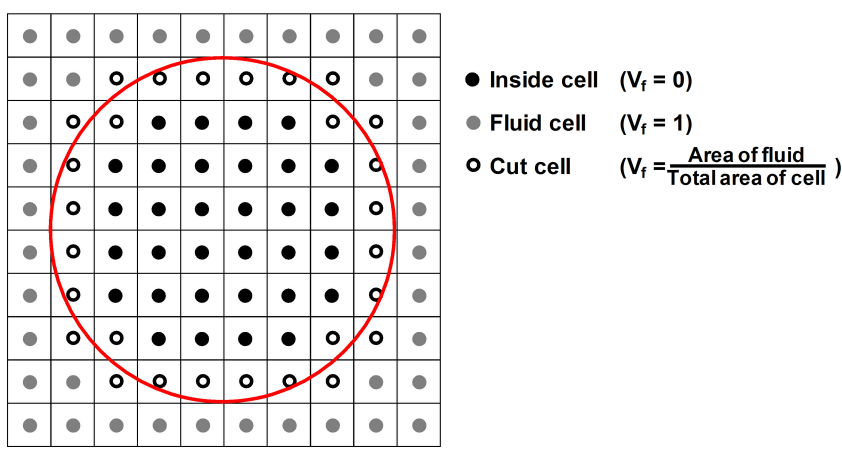

Figure 2. Concept of immersed boundary method of lowresolution LES on Cartesian grids. conducted by Tanino and Nepf [2008] and high-resolution LES simulations of Stoesser et al. [2010] are used. The high-resolution LES of Stoesser et al. [2010] resolved the individual cylinders directly through extremely fine grids, and the results were thoroughly validated and considered experiments for the purpose of this study. Also, supplementary laboratory experiments were carried out in the hydraulics laboratory at Georgia Tech for additional vegetation densities and stem Reynolds numbers. Overall, seven different vegetation densities, $\phi$, are investigated, with $\phi$ defined as follows:

$$
\phi=m \pi \frac{D^{2}}{4},
$$

in which $m$ is the number of stems per unit area and $D$ is the stem diameter.

[14] In addition to the vegetation density, different stem Reynolds numbers, $R e_{D}$, are investigated. $R e_{D}$ is defined as

$$
R e_{D}=\frac{u_{\text {bulk }} D}{\nu},
$$

in which $u_{\text {bulk }}$ the bulk fluid velocity, i.e., the discharge divided by the flow area, and $\nu$ is the kinematic viscosity of the working fluid. Specific information of the experimental cases is provided in the following sections and an overview is given in Table 2.

\subsection{Numerical Simulations by Stoesser et al. [2010]}

[15] The high-resolution LES were performed in analogy to the experimental investigations undertaken by Liu et al. [2008]. They placed a matrix of rigid cylinders in a staggered arrangement into a rectangular flume and carried out detailed LDA measurements. The distance between neighboring cylinders in both stream- and spanwise directions is $s=10 D(\phi=0.016)$. The Reynolds number based on the bulk velocity, $u_{\text {bulk, }}$ and the cylinder diameter, $D=$ $0.00635 \mathrm{~m}, R e_{D}$, is $\sim 1340$. The validation of the highresolution LES is described in detail by Stoesser et al. [2010]. They reported that their high-resolution LES reproduced the experiments quite accurately in terms of first- and second-order statistics. After successful validation of this case, Stoesser et al. [2010] expanded the parameter range by performing simulations for two additional vegetation densities, $\phi=0.063(s=5 D)$ and $\phi=0.251(s=2.5 D)$, and for additional stem Reynolds numbers, $R e_{D}=500,250$, and 125 for some cases. In total, eight high-resolution LES cases are available for comparison with RANS and low-resolution 
Table 2. Summary of Experimental Cases in Present Study and Predicted Bulk Drag Coefficients From Low-Resolution LES

\begin{tabular}{|c|c|c|c|c|c|c|c|c|}
\hline \multirow[b]{2}{*}{ Study } & \multirow[b]{2}{*}{ Stem Array } & \multicolumn{5}{|c|}{ Experimental Cases } & \multicolumn{2}{|c|}{ Low Resolution LES } \\
\hline & & $\phi$ & $\operatorname{Re}_{D}$ & $H / D$ & $C_{D}$ & $C_{D}$ (Cylinder Only) & $C_{D}$ & Percent Error \\
\hline \multirow[t]{8}{*}{ Stoesser et al. [2010] } & \multirow[t]{8}{*}{ Staggered } & 0.016 & 500 & 10.22 & 1.28 & 1.17 & 1.23 & 3.8 \\
\hline & & 0.016 & 1340 & 10.22 & 1.31 & 1.23 & 1.06 & 18.9 \\
\hline & & 0.063 & 125 & 10.22 & 2.05 & 1.96 & 1.79 & 12.8 \\
\hline & & 0.063 & 250 & 10.22 & 1.88 & 1.82 & 1.62 & 14.1 \\
\hline & & 0.063 & 500 & 10.22 & 1.79 & 1.75 & 1.55 & 13.8 \\
\hline & & 0.063 & 1340 & 10.22 & 1.72 & 1.68 & 1.53 & 10.7 \\
\hline & & 0.251 & 500 & 10.22 & 3.48 & 3.46 & 3.84 & 10.1 \\
\hline & & 0.251 & 1340 & 10.22 & 3.21 & 3.19 & 3.86 & 20.4 \\
\hline \multirow[t]{2}{*}{ Georgia Tech Experiments } & \multirow[t]{2}{*}{ Staggered } & 0.022 & 1700 & 13.4 & 1.43 & - & 1.16 & 18.9 \\
\hline & & 0.087 & 695 & 16.5 & 2.02 & - & 1.90 & 5.8 \\
\hline \multirow[t]{8}{*}{ Tanino and Nepf [2008] } & \multirow[t]{8}{*}{ Randomly } & 0.091 & 170 & 24.62 & 2.14 & - & - & - \\
\hline & & 0.091 & 500 & $15-34$ & $1.35-2.09$ & - & 2.00 & - \\
\hline & & 0.091 & 1340 & $15-34$ & $1.32-2.00$ & - & 1.95 & - \\
\hline & & 0.150 & 193 & 20.89 & 3.03 & - & - & - \\
\hline & & 0.150 & 263 & 21.38 & 2.86 & - & - & - \\
\hline & & 0.150 & 363 & 22.69 & 2.72 & - & - & - \\
\hline & & 0.150 & 500 & $15-34$ & $1.96-2.83$ & - & 2.49 & - \\
\hline & & 0.150 & 1340 & $15-34$ & $1.78-2.59$ & - & 2.44 & - \\
\hline
\end{tabular}

LES. Figure 3a depicts the numerical setup of the high-resolution LES and the grid resolution of each high-resolution simulation is detailed in Table 3.

\subsection{Laboratory Experiments by Tanino and Nepf [2008]}

[16] The laboratory experiments by Tanino and Nepf [2008] investigated the drag exerted by randomly distributed, rigid, emergent, circular cylinders of uniform diameter on the flow. The authors covered the following vegetation densities $(\phi=0.091,0.15,0.20,0.27$, and 0.35$)$ and a fairly wide range of stem Reynolds numbers, $R e_{D}=25-685$. They provide the bulk drag coefficient in an equation form (i.e., equation (5) in their paper), which is used herein to attain the corresponding drag coefficients. In the numerical study presented here, two densities, i.e., $\phi=0.091$ and 0.15 , are selected for RANS and low-resolution LES evaluation. In addition, one additional flow with $R e_{D}=1340$ is

(a)

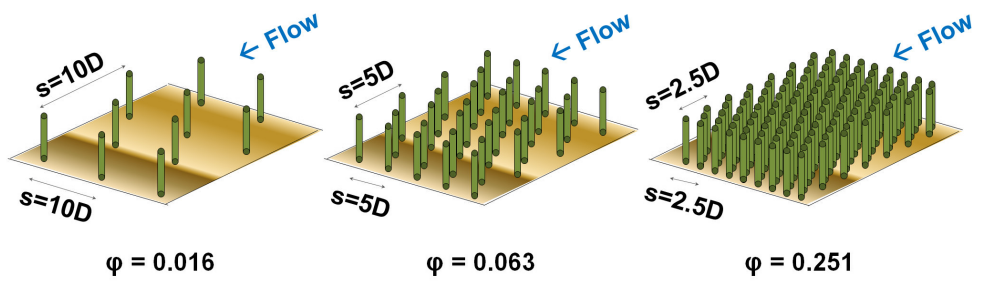

(b)

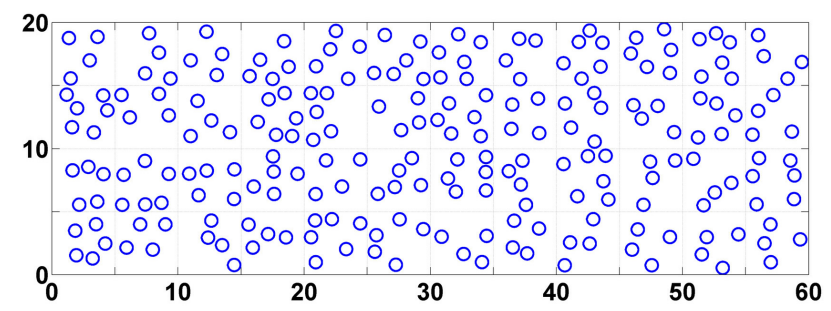

(c)

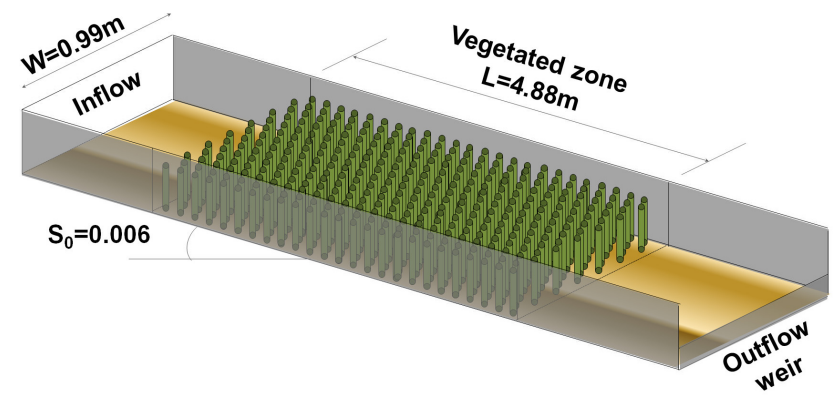

Figure 3. Setup of test cases used in this study; (a) high-resolution LES of Stoesser et al. [2010], (b) sketch of experiments by Tanino and Nepf [2008], and (c) experiments at Georgia Tech. 
Table 3. Grid Resolution of the Present Simulations and for Comparison of the High-Resolution LES of Stoesser et al. [2010]

\begin{tabular}{cccccc}
\hline \multicolumn{2}{c}{ Cases } & & \multicolumn{3}{c}{ Total Number of Gridpoints } \\
\cline { 1 - 2 } \cline { 5 - 6 }$\phi$ & $R e_{D}$ & & High-Resolution LES & RANS & Low-Resolution LES \\
\hline 0.016 & 500 & $7,564,000$ & 275 & 534,681 \\
& 1340 & & $11,604,640$ & 275 & 534,681 \\
0.063 & 125 & $12,297,600$ & 275 & 534,681 \\
& 250 & $12,297,600$ & 275 & 534,681 \\
& 500 & $12,297,600$ & 275 & 534,681 \\
0.251 & 1340 & $22,994,560$ & 275 & 534,681 \\
& 500 & $9,963,008$ & 891 & 534,681 \\
& 1340 & $29,514,240$ & 891 & 534,681 \\
\hline
\end{tabular}

tested for both densities with the assumption that the relationship provided by Tanino and Nepf can be extrapolated to higher stem Reynolds numbers, i.e., $R e_{D}>685$. The numerical setup of these laboratory experiments utilizes MATLAB's (The MathWorks, Inc., www.mathworks.com) random number generator, with which the distribution of the cylinders is determined for the two densities (e.g., $\phi=0.15$ in Figure $3 b$ ).

\subsection{Supplementary Laboratory Experiments at Georgia Tech}

[17] Additional experiments were conducted in the Georgia Tech hydraulic laboratory in an $8 \mathrm{~m}$ long, $0.99 \mathrm{~m}$ wide, $0.4 \mathrm{~m}$ deep flume that has a fixed bottom slope of $S_{0}=$ 0.006 (Figure 3c). Two different vegetation densities, $\phi=0.022(s=8.5 D)$ and $\phi=0.087(s=4.25 D)$ were emulated using thin, wooden dowels of diameter $D=0.01 \mathrm{~m}$, which were arranged in a staggered fashion. The dowels covered a length of $4.88 \mathrm{~m}$ of the flume, whereas the exit and entrance section featured coarse gravel to obtain a smoother flow transition into and out of the vegetation section. Different flow scenarios were investigated, mainly to determine the uniform flow depth for seven different discharges at two different vegetation densities, i.e., $\phi=$ 0.022 and $\phi=0.087$. Water surface profiles were measured with a point gage along the centerline of the flume at intervals of $0.3 \mathrm{~m}$. The precision of the point gage was $1 / 10 \mathrm{~mm}$. A flexible tailgate allowed for setting different downstream water depths. The uniform flow depth was found from the intersect of M1 and M2 water surface profiles for the same discharge. A regression analysis of the measured data was used to determine the slope of the two profiles. Once the uniform flow depth was determined, the tailgate was adjusted accordingly and flow uniformity was confirmed by repeating the water surface profile measurements. The stem Reynolds number based on dowel diameter, $R e_{D}$ varied between 1675 and 1750 and 600 to 695 for the $\phi=0.022$ and $\phi=0.087$ cases, respectively. Two flows for each vegetation density were selected to supplement the high-resolution LES and the experimental data of Tanino and Nepf [2008].

\section{Results and Discussion}

\subsection{The Effect of $R e_{D}$ and $\varphi$ on the Bulk Drag Coefficients}

[18] The selected experiments (and simulations) are analyzed first for the bulk drag coefficient, $C_{D}$ in particular for the effect of stem Reynolds number and vegetation density.
This is summarized in Table 2. In the bulk drag coefficient of the laboratory experiments conducted at Georgia Tech, $C_{D}$, is computed by equaling the integral shear force as a result of gravity, $F_{g}$ and the sum of the drag forces exerted by the emergent vegetation, $F_{D}$,

$$
\begin{gathered}
F_{g}=F_{D}, \\
\rho g H S_{0}=M \frac{1}{2} \rho C_{D} H D u_{\text {bulk }}^{2}, \\
C_{D}=\frac{g S_{0}}{M \frac{1}{2} D u_{\text {bulk }}^{2}},
\end{gathered}
$$

in which $g$ is the acceleration due to gravity $\left(=9.81 \mathrm{~m} \mathrm{~s}^{-2}\right)$, $S_{0}$ is the bottom slope of the channel, and $M$ is the total number of individual stems in the measurement section.

[19] In this formulation, the channel bottom and channel sidewall friction is assumed to be negligibly small, which is a common assumption [Ferreira et al., 2009; Kothyari et al., 2009; Tanino and Nepf, 2008]. An advantage of including the high-resolution LES as additional experiments is that this assumption can be proven directly, because Stoesser et al. [2010] have provided the contributions of cylinder drag and bed shear drag to the total flow resistance. The effect of bed friction on the bulk drag will be discussed later.

[20] The dependence of the bulk drag coefficient on $\operatorname{Re}_{D}$ and $\phi$ is shown in Figure 4. Darker symbols in the figure represent denser vegetation. The symbol shape varies according to the data source, i.e., diamonds represent the data of Stoesser et al. [2010], circles represent the data of Tanino and Nepf [2008], and triangles represent the data of the additional experiments carried out at Georgia Tech. From Figure $4 \mathrm{a}$ it is apparent that $C_{D}$ is a function of both $R e_{D}$ and $\phi$. The effect of the stem Reynolds number is less significant than the effect of vegetation density, which is better illustrated in Figure 4b. In Figure $4 \mathrm{~b}$ a decrease in the stem Reynolds number is indicated by solid arrows and at low vegetation density the effect of Reynolds number disappears, which is consistent with experiments of flow around an isolated cylinder. The $C_{D^{-}}$-values obtained from the laboratory experiments carried out as part of this study are consistent with and complement previous observations [Ferreira et al., 2009; Kothyari et al., 2009; Tanino and Nepf, 2008]. Noteworthy is the fact that the vegetation arrangement, i.e., staggered versus random, does not seem to affect the value of the bulk drag coefficient. This is easily observable when comparing the drag coefficient obtained by Tanino and Nepf, i.e., gray circles, with the $C_{D}$ found from the Georgia Tech experiments (i.e., gray triangles). Both experiments featured a vegetation density of $\phi \approx 0.09$.

\subsection{The Importance of A Priori Knowledge of Drag Coefficients on Flow Resistance Predictions Using RANS}

[21] As mentioned above, RANS models employ a vegetation closure model that includes an empirical coefficient, i.e., the bulk drag coefficient. In this study, two sets of RANS simulations are performed to investigate the importance of a priori knowledge of the drag coefficient and to 
(a)

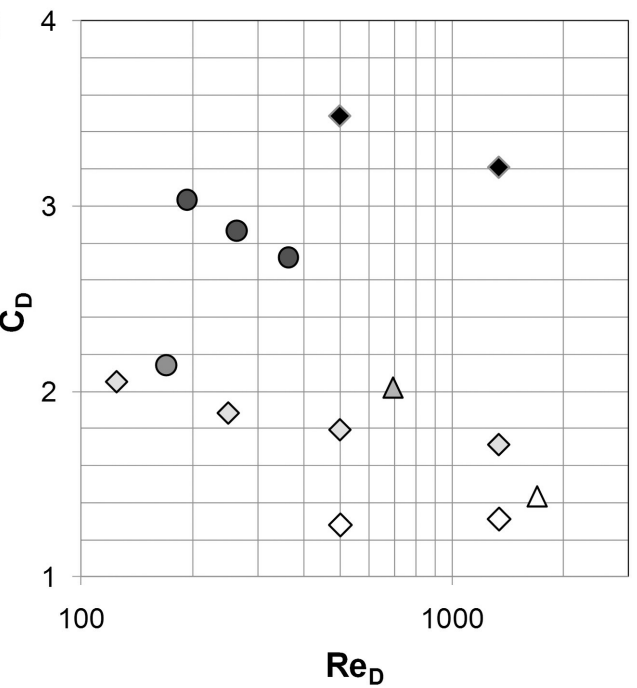

(b)

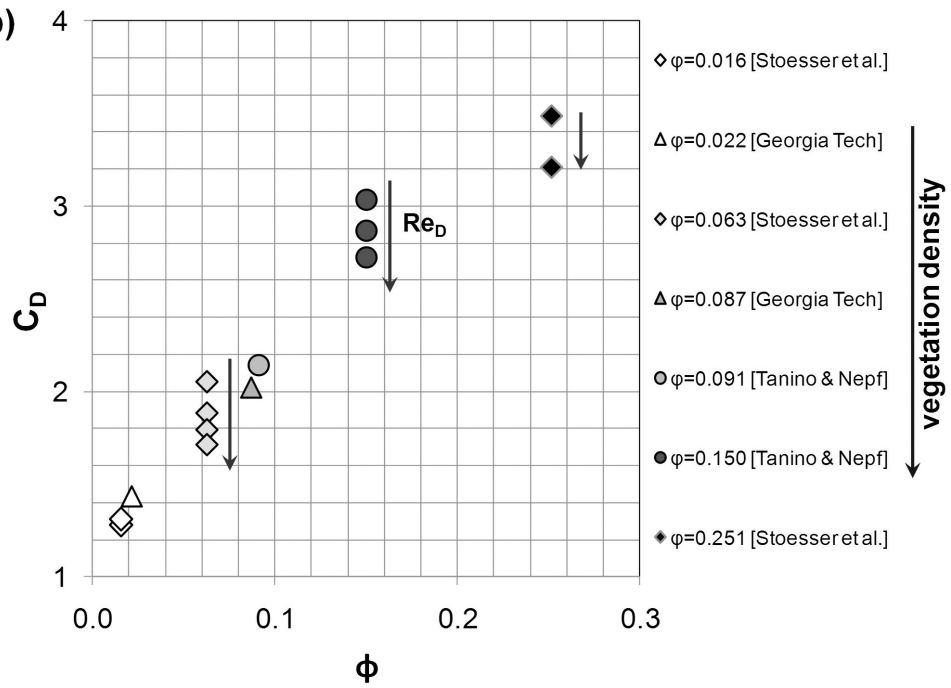

Figure 4. The effect of (a) $R e_{D}$ and (b) $\phi$ on the bulk drag coefficient.

quantify its influence on the headloss in the system; the first set of simulations uses known drag coefficients (from the corresponding experiment or from the formula to calculate $C_{D}$ suggested by Tanino and Nepf [2008], and the other set of simulations uses an assumed drag coefficient of unity (commonly used when there is a lack of knowledge of the exact $\left.C_{D}\right)$. For the quantification of flow resistance, the normalized global bed shear stress, i.e., $\tau_{0} /\left(u_{\text {bulk }}^{2} \rho\right)$, is used. The value obtained from the RANS simulation $\left(\tau_{0}\right.$ calculated as $\tau_{0}=d p / d x \times H$ of each available experiment) is then compared with the one measured ( $\tau_{0}$ calculated as $\left.\tau_{0}=\rho \times g \times H \times S_{0}\right)$. Figure 5a compares the first set of RANS simulations using known bulk drag coefficients with experimental values and the data almost collapses on the $45^{\circ}$ line that indicates a perfect match. For the densest vegetation case, i.e., $\phi=0.251$, RANS slightly underestimates the flow resistance, though the exact cylinder drag coefficient was provided from the high-resolution LES. This should

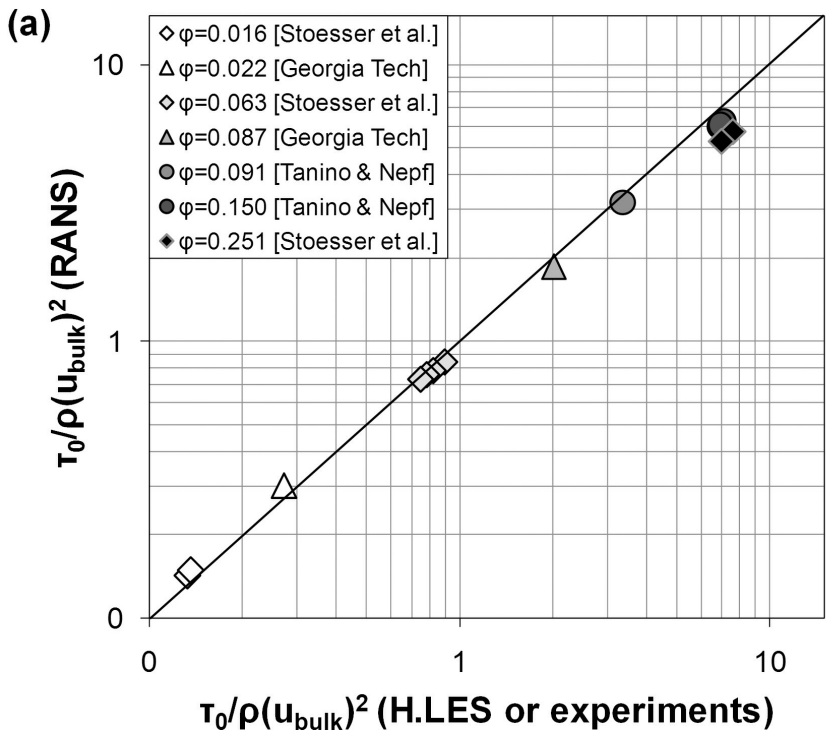

be attributed to the fact that in RANS strong stream- and spanwise velocity gradients that are prevailing in the flow with the highest vegetation density are not reproduced.

[22] The RANS simulations using a constant bulk drag coefficient of $C_{D}=1.0$ generally underestimate the flow resistance (Figure $5 b$ ). The underestimation of flow resistance is more severe with increasing vegetation density, and the maximum error is found to be almost $78 \%$ for the cases with $\phi=0.251$. However, the lower density cases exhibit better agreement, especially in the cases of $\phi=0.016$ and $\phi=0.022$. This is owing to the fact that the flow through vegetation at low vegetation density is similar to the flow around an isolated cylinder [Stoesser et al., 2010], in which the drag coefficient converges to unity over a wide range of stem Reynolds numbers.

[23] Overall, a priori knowledge of the bulk drag coefficient is found to be important when employing a vegetation closure model in RANS simulations. This study shows that

Figure 5. Predictions of global shear stress using RANS with (a) a priori known $C_{D}$ and (b) $C_{D}=1.0$. 
if a drag coefficient of unity was used for simulations of the flow through emergent vegetation, the headloss in the system would be largely underpredicted especially at high vegetation density or low stem Reynolds numbers, respectively.

\subsection{The Contribution of Bed Friction}

[24] When determining the bulk drag coefficient from experiments, the bed friction is assumed to be negligibly small. Hence, so determined $C_{D}$-values theoretically include both vegetation drag and bed friction. What follows is that a RANS simulation that uses an experimentally determined bulk drag coefficient accounts for the bed friction twice and tends to overestimate the global bed shear stress, $\tau_{0}$. In the high-resolution LES of Stoesser et al. [2010] the percentage contribution of bed friction to the overall drag was calculated directly from the high-resolution data. Figure $6 \mathrm{a}$ illustrates the contribution of bed friction as a function of vegetation density and stem Reynolds number. The LES data are regressed for each available stem Reynolds number using a power law. For both data sets the squared regression coefficient is $R^{2}=0.9998$. As with the bulk drag coefficient, the bed friction shows a greater dependency on the vegetation density than on the stem Reynolds number, and its contribution increases exponentially for vegetation densities $<0.1$. This information is used to correct the bulk drag coefficient obtained from the experiments and a third set of RANS simulations is carried out, but only for the physical experiments. Figure $6 \mathrm{~b}$ presents the results of the third set of RANS simulations, again directly comparing the simulated global shear stress with the measured one. It is seen that the already good match is not necessarily improved further. Only minimal improvements are observed for the low density experiment (white triangle), and for the higher vegetation density flows of Tanino and Nepf [2008] the difference is not noticeable. However, given the exponential increase in bed friction contribution at decreasing vegetation density, the assumption of negligible bed friction may be invalid for vegetation densities lower than the ones reported herein.

\subsection{Evaluation of the Low-Resolution LES Approach}

[25] Above simulations have demonstrated the importance of a priori knowledge of the bulk drag coefficient in RANS models that feature a common cylinder drag-based vegetation closure. For uniform flow through emergent vegetation in which the vegetation covers the entire channel width, the relationships given by Tanino and Nepf [2008] can be used to calculate the unknown $C_{D}$-value. The so obtained $C_{D}$ can then be plugged into a RANS vegetation closure to predict reliably the flow resistance due to emergent vegetation. Figure 7 a compares the $C_{D}$ 's of the present study with the range of $C_{D}$ 's computed from Tanino and Nepf's equation. It appears that the measured $C_{D}$-values are consistently at the higher end of the range of the $C_{D}$ 's computed with Tanino and Nepf's equation. This could be attributed to the fact that Tanino and Nepf's experiments were carried out at gradually varying flow, while the data used herein stem from experiments and simulations under uniform flow conditions. Nevertheless, the so calculated $C_{D^{-}}$-values match observed values under uniform flow conditions quite well, regardless of the stem Reynolds number.

[26] In practical flows, however, the vegetation may not be distributed uniformly over the entire channel width or length in question. For instance, vegetation on floodplains may grow in heterogeneous patches and the drag of individual plants may vary largely within the patch. For such engineering applications the high-resolution LES is unfeasible because of the high computational costs; hence, it is not an alternative to RANS. A less expensive, low-resolution LES may overcome the dependency on a priori knowledge of $C_{D}$ of RANS vegetation closures and at the same time decreases considerably the enormous computational effort of a fully resolved LES. The low-resolution LES of the present study are performed with a 10 times finer grid (in each flow direction) than the RANS and with a 20 times coarser grid (in the (a)

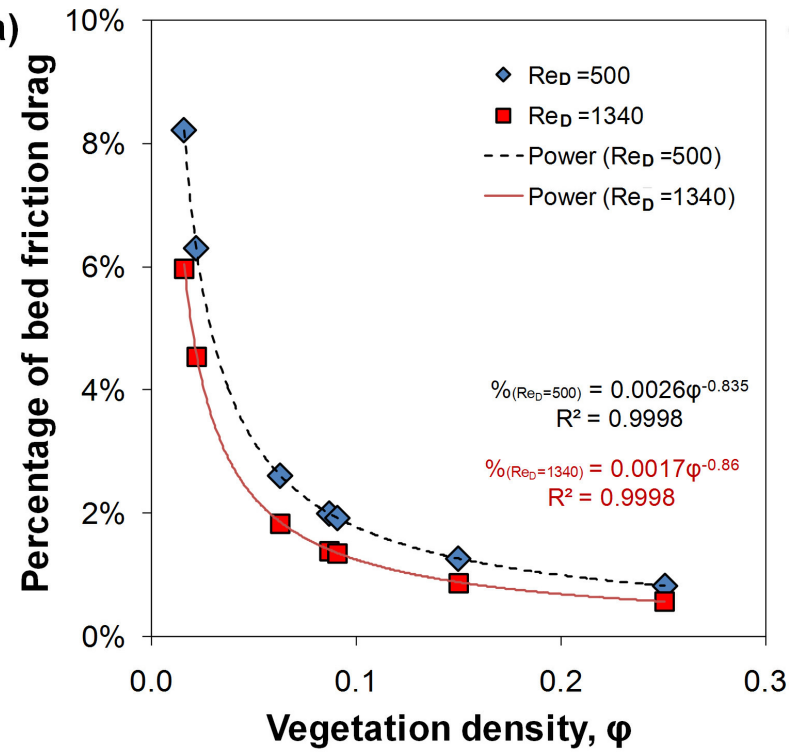

(b)

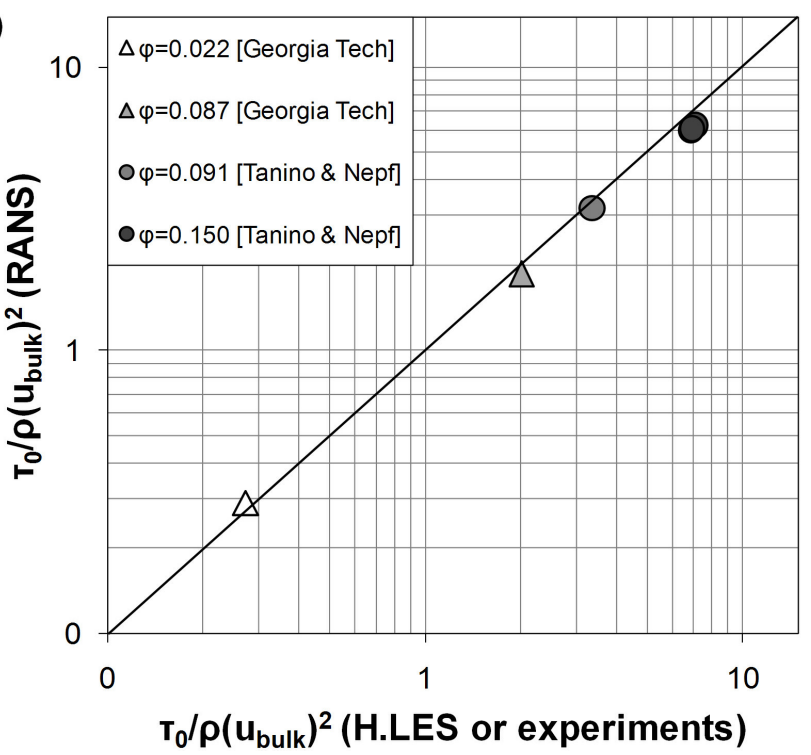

Figure 6. (a) Percentage of bed shear stress as a function of vegetation density and (b) comparison of global shear stress between experiments and RANS. 
(a)

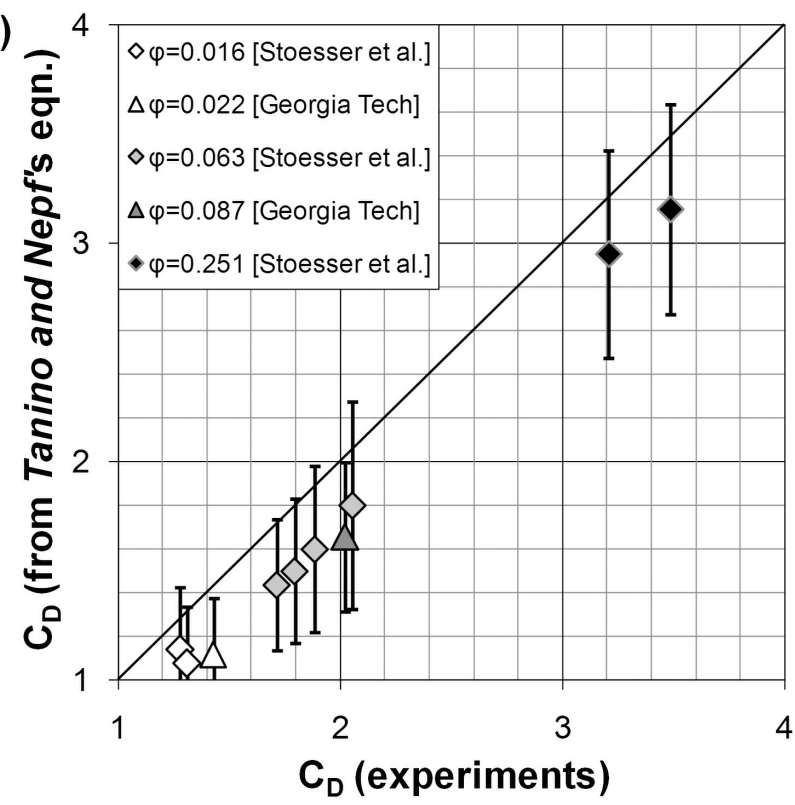

(b)

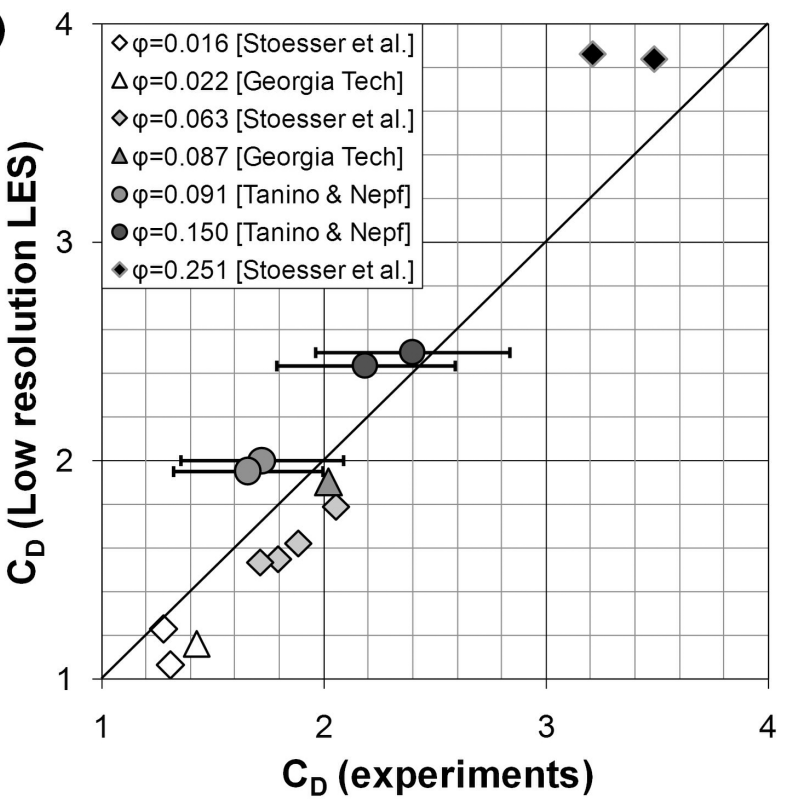

Figure 7. Comparison of drag coefficients from experiments with (a) $C_{D}$ from Tanino and Nepf's equation and (b) $C_{D}$ predicted by low-resolution LES.

horizontal) than the LES (see Table 3). If successful, the method of low-resolution LES could be used to provide drag coefficients for more complex vegetation arrangements.

[27] The total drag in the low-resolution LES is calculated from the pressure gradient that drives the flow as $F_{D, \text { total }}=(d p / d x) \times \mathrm{Vol} \times(1-\phi)$. The drag coefficient is then computed from $C_{D}=2 F_{D \text {,total }} /\left(u_{\text {bulk }}^{2} \times M \times H \times D\right)$. The following vegetation densities are considered, $\phi=$ $0.016,0.022,0.063,0.087,0.091,0.150$, and 0.251 , and the predicted bulk drag coefficients are summarized in Table 2. A comparison of calculated $C_{D}$ 's with the measured ones is plotted in Figure $7 \mathrm{~b}$, showing an overall reasonably good agreement with the experimental cases except for the densest vegetation case with $\phi=0.251$. The percentage error is in the range of $1 \%-20 \%$ (see also Table 2). It is obvious that the low-resolution LES generally underestimates the drag coefficient, in particular for the cases in which the vegetation is arranged in a regular (staggered) way. For the randomly arranged vegetation by Tanino and Nepf [2008], the low-resolution LES predicts the bulk drag coefficient near the higher end of the range provided by Tanino and Nepf's equation. This suggests fairly good agreement (see discussion of Figure $7 \mathrm{a}$ ). The $\phi=0.251$ case of the high-resolution LES shows that the drag coefficient is overestimated, and for the higher stem Reynolds number the overestimation is $\sim 20 \%$. In the low-resolution LES approach the grid is too coarse to reliably compute the distribution of pressure on the cylinder, and a way of assessing the accuracy of the LES method is to investigate the size and strength of the recirculation behind each cylinder.

[28] Figures 8 and 9 present contours of the streamwise velocity together with streamlines for the case of $\phi=$ 0.087 and $\phi=0.251$ at half water depth (i.e., $Z / H=0.5$ ) for both the high- and low-resolution LES. The results of the low-resolution LES for the $\phi=0.087$ case reasonably match the high-resolution LES in terms of the velocity gradients (see the top row of Figure 8) and in terms of the recirculation region (the bottom row of Figure 8). The length of the recirculation zone is minimally shorter in the low-resolution LES of $\phi=0.087$, leading to minimal underprediction of the drag coefficient (see Table 2). For the high density case, i.e., $\phi=0.251$, the match is obviously not as good, and the velocity gradients (top row of Figure 9) as well as the size of the recirculation zone (the bottom row of Figure 9) are predicted erroneously by the low-resolution LES (here overprediction of the length). Additionally, because very strong streamwise velocity gradients occur, the simulation suffers from numerical wiggles, which are a result of the central differencing scheme on a coarse grid. A more quantitative comparison of the timeaveraged streamwise velocity is carried out by extracting profiles along two stream- and spanwise lines (Figures 10a and $10 \mathrm{~b}$, respectively, here only for the $\phi=0.087$ case). The time-averaged velocity from both the high- and lowresolution LES exhibit a pretty good overall match. The low-resolution LES is able to reproduce both streamwise and spanwise velocity gradients quite accurately. The stagnation point and the recirculation region behind the cylinder are resolved adequately, and the mismatch in recirculation length is obvious from profile $\times 1$.

[29] Figure 11 presents vertical profiles of the spatially averaged streamwise velocity (normalized with the free stream velocity, $u_{0}$, i.e., the magnitude of the velocity in the spatially averaged velocity profile away from the bed) from the low-resolution LES, RANS, and, for comparison, high-resolution LES for three cases with $\phi=0.016,0.063$, and 0.251 at $R e_{D}=1340$ (Figures $11 \mathrm{a}-11 \mathrm{c}$ ). It is remarkable that even though the RANS simulations are unable to capture all the details of the flow, the vertical velocity profiles of RANS match the spatially averaged velocity profile of the high-resolution LES quite well. This is true regardless of vegetation densities. Also, RANS is able to predict the decrease in bottom boundary layer thickness as vegetation density increases. However, the RANS simulated 
(a)
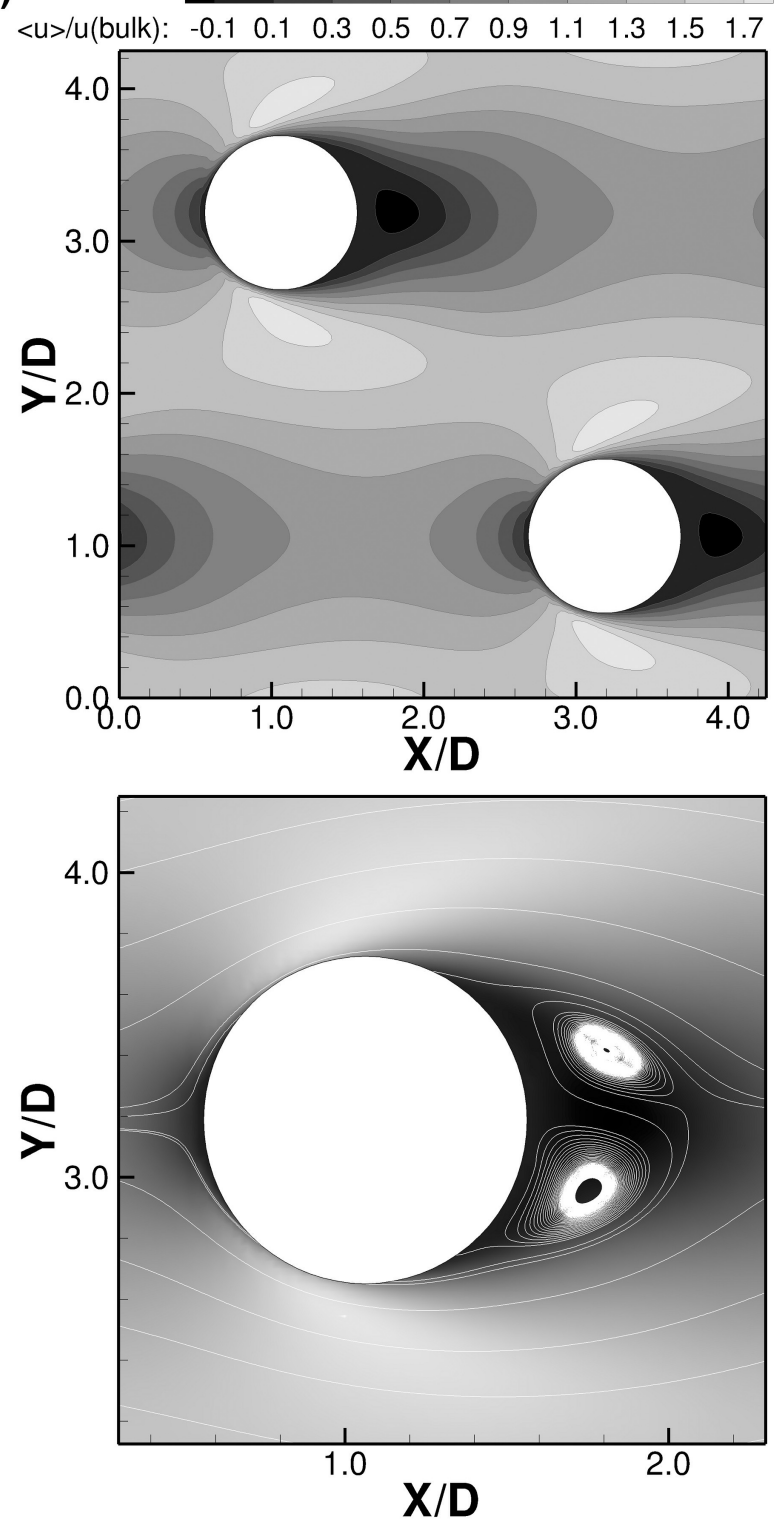

(b)
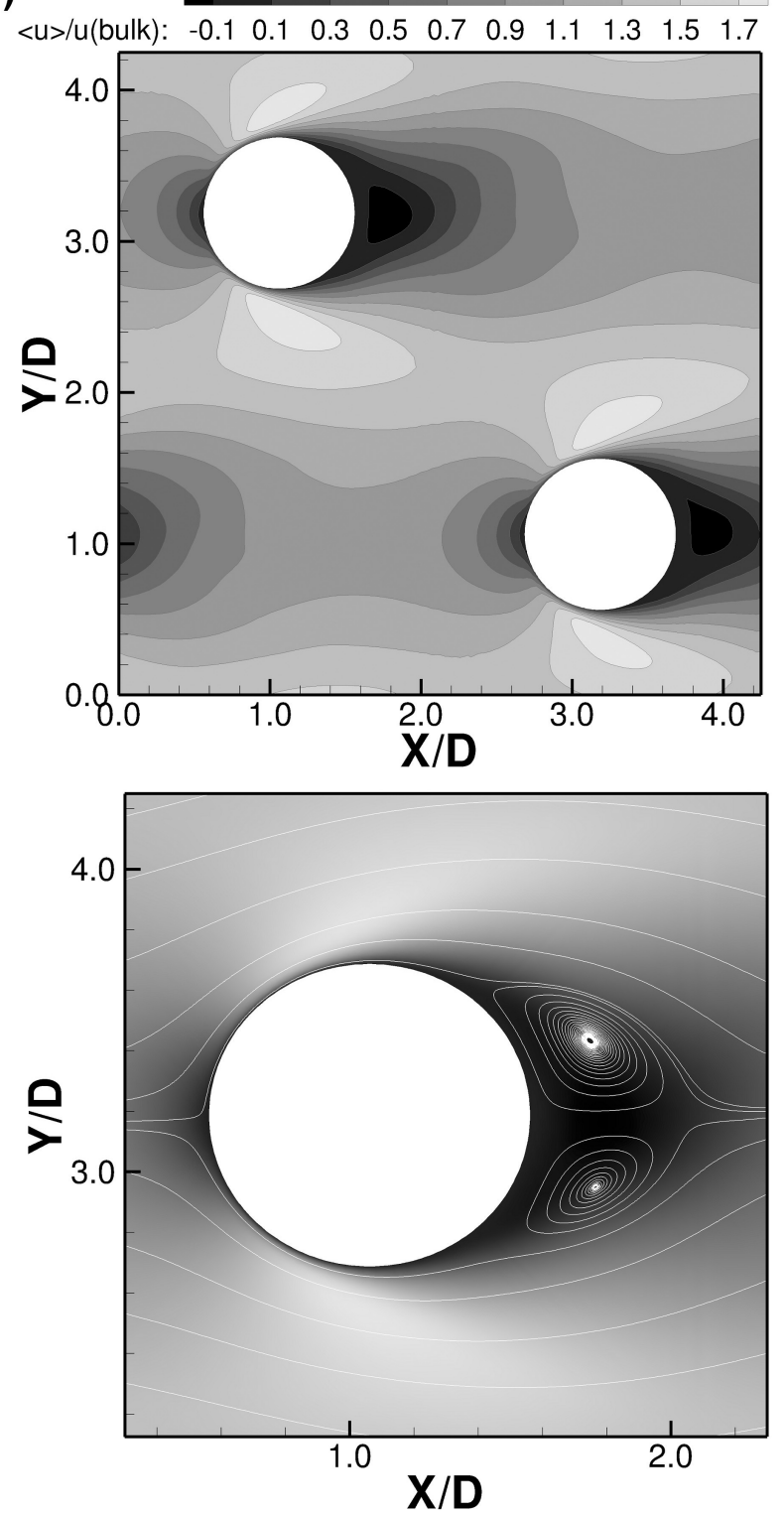

Figure 8. Contours of time-averaged velocity (top row) and streamlines around one cylinder (bottom row) of the $\phi=0.087$ case simulated by (a) low- and (b) high-resolution LES.

profiles show some deviations near the bed, where RANS computes a thicker boundary layer and is unable to predict the velocity bulge. Both features are a result of secondary currents [see Stoesser et al., 2010] that are transporting high-momentum flow to the channel bed, and since a RANS model that uses a drag force vegetation closure does not resolve the flow around individual cylinders it cannot predict such flow features. The low-resolution LES is able to resolve reasonably well the velocity gradients, a wake behind the cylinder, and secondary currents. As a result, the spatially averaged velocity profiles are in relatively good agreement with the high-resolution LES, including boundary layer thickness and velocity bulge.

[30] A more detailed comparison of individual velocity profiles of low- and high-resolution LES is presented in Figure 12. This is done for the cases in which the low-resolution
LES exhibits the best (i.e., $\phi=0.016$ ) and the worst (i.e., $\phi=0.251$ ) performance in terms of predicting the bulk drag coefficient. Five velocity profiles around the cylinders are selected, and their location in the flow is indicated in the included sketch. The comparison of the profiles confirm the statements made above, i.e., the existence of streamand spanwise gradients, that are particularly large in the $\phi=0.251$ case. For $\phi=0.016$, all of the profiles predicted by low-resolution LES match the high-resolution LES quite well, except near the bed at location 1, which is very close to the cylinder. Here the lower grid resolution prevents a better match. The overall good match is reflected in the small discrepancy in bulk $C_{D}$. For the high vegetation density case, the match between low- and high-resolution LES is quite poor along several profiles, in particular for profiles two and six. Apparently, the low-resolution LES is unable 
(a)
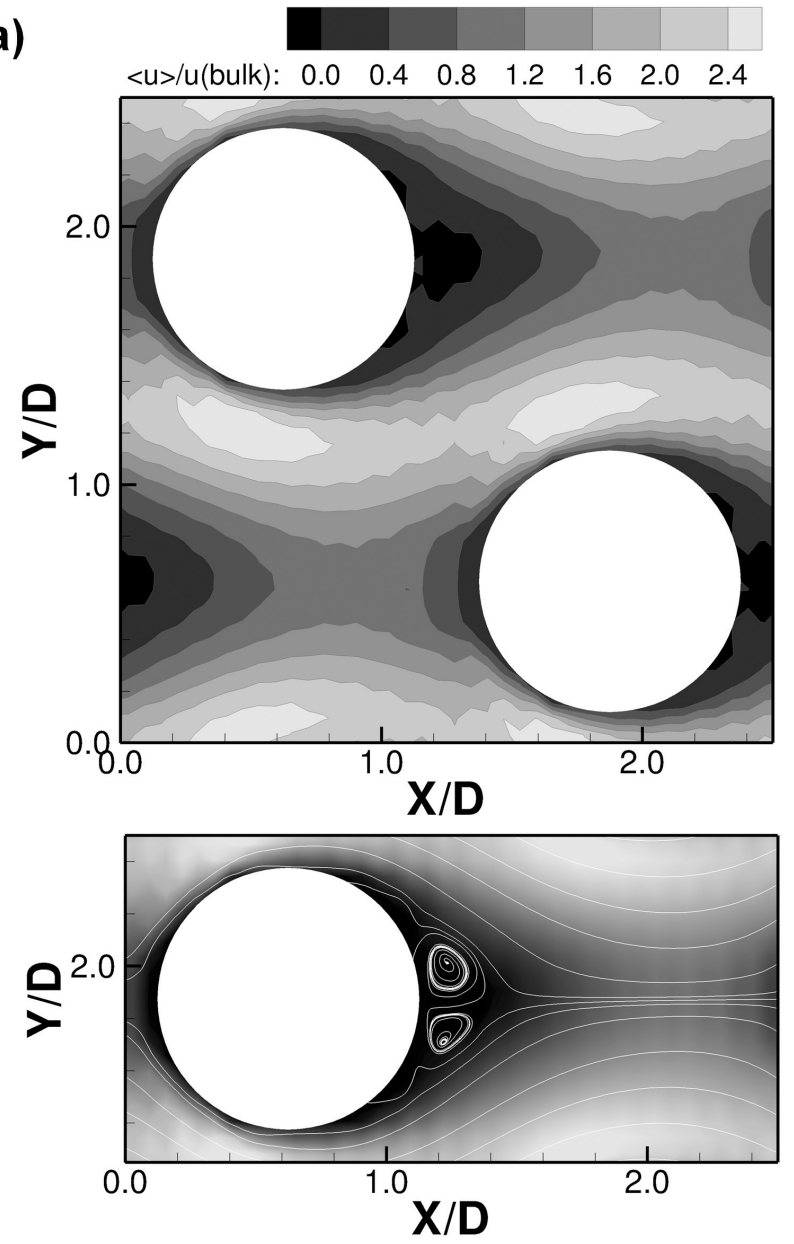

(b)
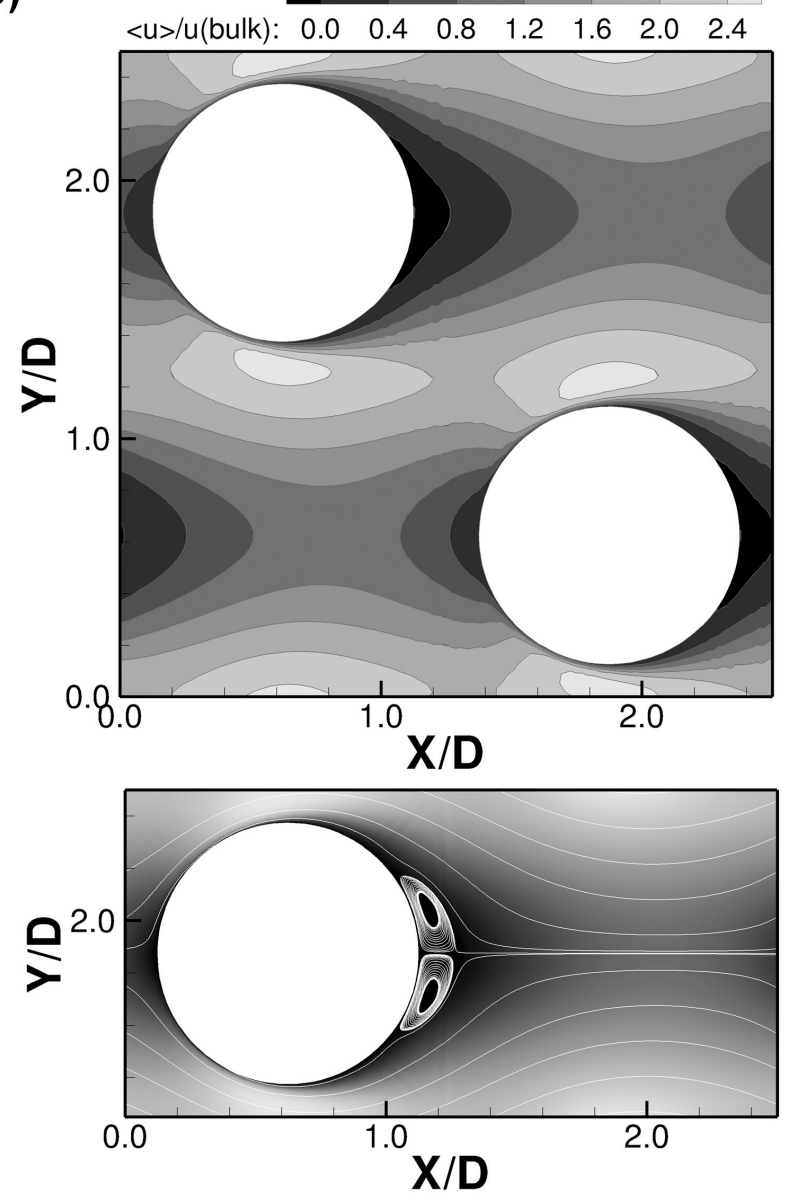

Figure 9. Contours of time-averaged velocity (top row) and streamlines around one cylinder (bottom row) of the $\phi=0.251$ case and $\operatorname{Re}_{D}=1340(Z / D=5)$ simulated by (a) low- and (b) high-resolution LES.

(a)

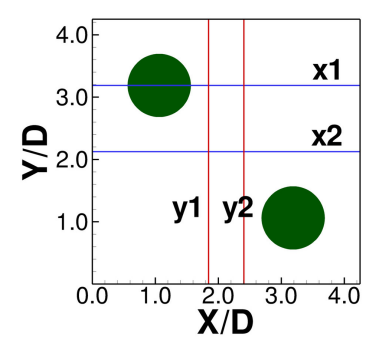

$<$ line location>

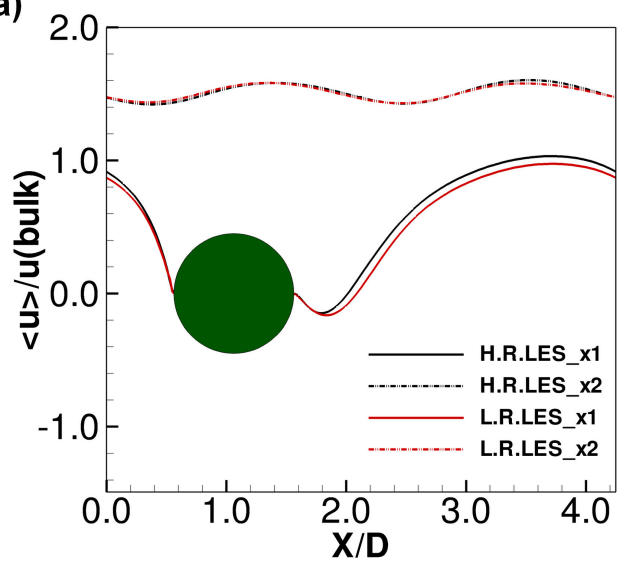

(b)

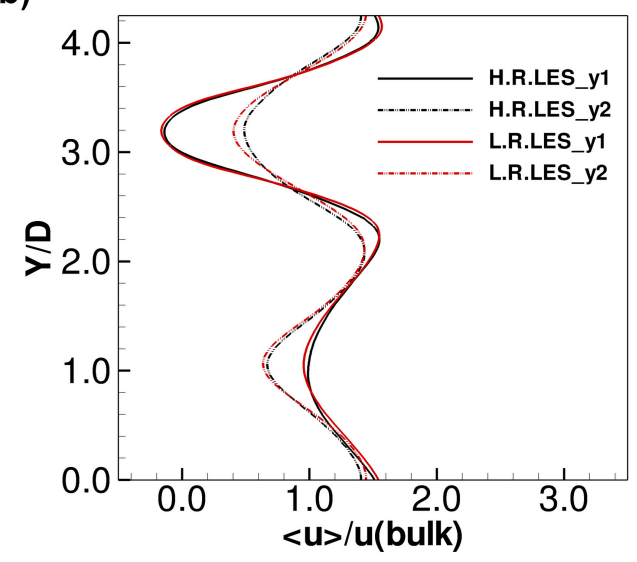

Figure 10. Profiles of time-averaged streamwise velocity from low- and high-resolution LES along selected lines of the $\phi=0.087$ case along (a) stream- and (b) spanwise directions. 
(a)

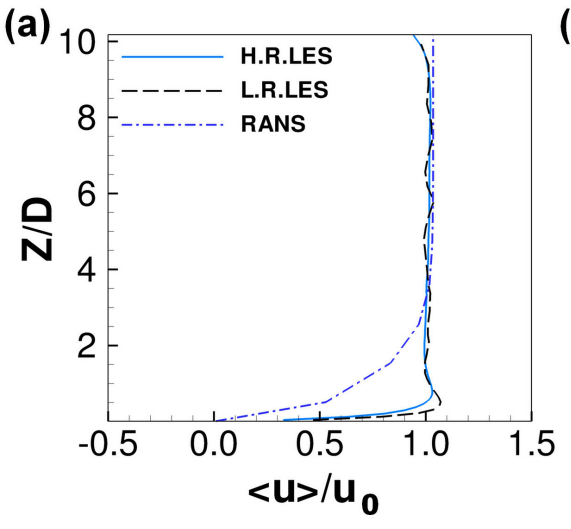

(b)

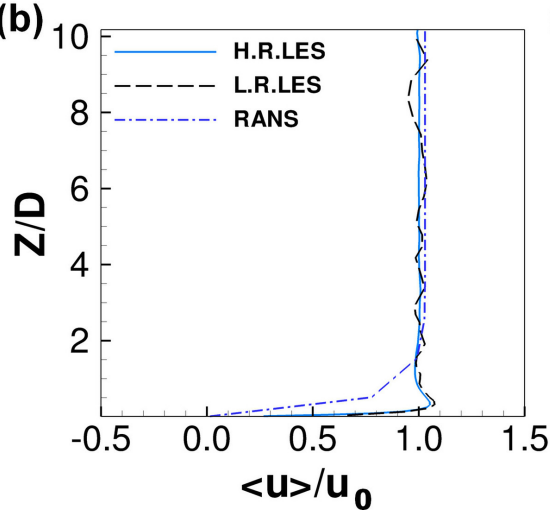

(c)

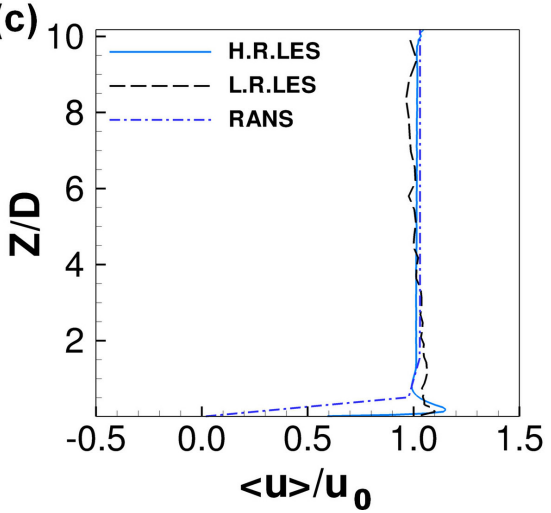

Figure 11. Vertical profiles of spatially averaged streamwise velocity for (a) $\phi=0.016$, (b) 0.063 , and (c) 0.251 at $R e_{D}=1340$.

to accurately reproduce velocity gradients, clearly overestimates the recirculation zone (see vertical 6), and even produces a rather unphysical profile at vertical 2. Interestingly, the spatially averaged velocity profile almost collapses onto the high-resolution LES profile (see Figure 11c). The rather large discrepancy in terms of the recirculation zone is directly reflected in the poor match of bulk drag coefficient.

[31] Finally, the computational costs of the different simulation approaches should be discussed. The RANS simulations were run on very coarse grids and a fully converged simulation for steady state took $<1$ min on a desktop computer. Both LES approaches are much more expensive in terms of computing time and demand sophisticated computer hardware. LES calculates the instantaneous flow and time-averaging requires sampling of each quantity at every time step over a certain averaging period. For example, the physical simulation time for 10 flow throughs (i.e., the time a fluid element requires to be convected through the computational domain) of the $\phi=0.016$ case took about 4600 and 140 CPU h by high- and low-resolution LES, respectively. For the simulations reported herein, $\sim 10$ flow
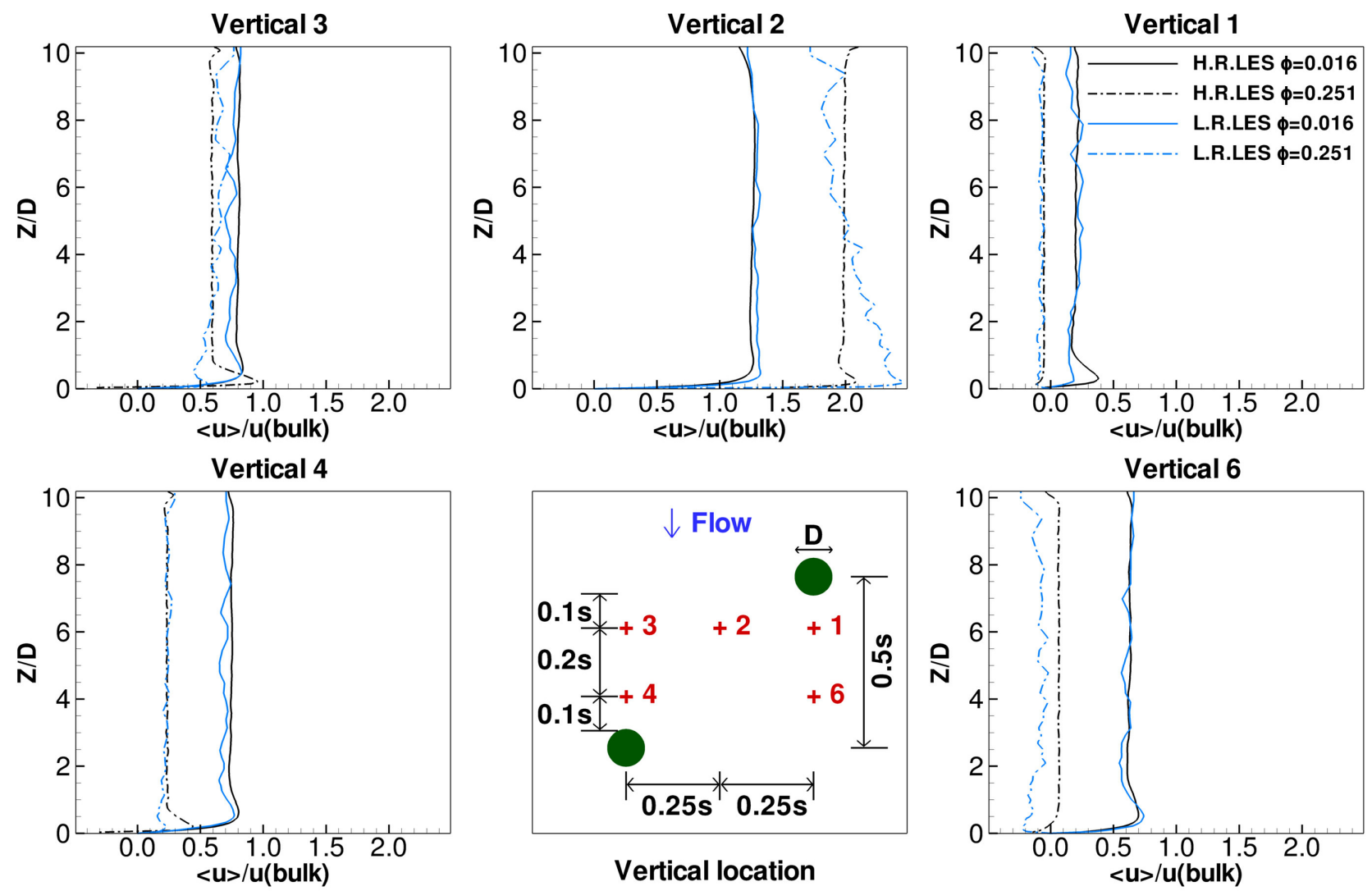

Figure 12. Time-averaged streamwise velocity profiles along five selected verticals for two densities of the $\phi=0.016$ and 0.251 cases at $R e_{D}=1340$ predicted by high- and low-resolution LES. 
Table 4. Grid Sensitivity Analysis of the Low-Resolution LES with $\phi=0.016$ and $R e_{D}=500$

\begin{tabular}{llccc}
\hline \multicolumn{1}{c}{ Numerical Strategy } & & $\begin{array}{c}\text { Total Number } \\
\text { of Gridpoints }\end{array}$ & $\begin{array}{c}\text { Number of Cells } \\
\text { Around a Cylinder }\end{array}$ & $C_{D}$ \\
\hline High-resolution LES & & $7,564,000$ & 240 & 1.28 \\
Low-resolution LES & (a) & $2,152,008$ & 64 & 1.27 \\
& (b) & 551,368 & 32 & 1.23 \\
& (c) & 144,648 & 16 & 1.26 \\
\hline
\end{tabular}

throughs are needed to develop the flow and another 30 flow throughs to collect reliable first-order statistics. For the high-resolution LES a 96 processor Linux cluster was needed, while the low-resolution LES was carried out on a 4 processor Linux workstation.

\subsection{Grid Sensitivity Analysis of Low-Resolution LES}

[32] As was shown above, the low-resolution LES method appears to work fairly well, at least for lower vegetation densities. The number of grid points chosen for the low-resolution LES is based mainly on efficiency (with upscaling in mind) and not necessarily on near wall LES resolution requirements. Therefore, a grid sensitivity study is carried out for the case of $\phi=0.016$ at $\operatorname{Re}_{D}=500$ in order to determine if the accuracy increases/decreases with an increase/decrease in grid resolution. Two more grids are generated, i.e., one grid (referred to as case (a) in the following) has a 4 times higher resolution than the original low-resolution grid (in the following case (b)), while the other grid resolution (case (c)) has 4 times less grid points than the original low-resolution grid (details are provided in Table 4). The number of grid points in the vertical is kept constant. The numbers of cut cells (see Figure 2) for the immersed boundary of one cylinder in a horizontal plane are 64, 32, and 16 for the cases (a), (b), and (c), respectively. The number of cut cells used in case (a) is still considerably less than the number of cells in the bodyfitted, curvilinear grid of the high-resolution LES (i.e., 240) to resolve one cylinder. The results in terms of the bulk drag coefficient of the grid sensitivity study are also provided in Table 4. Even the lowest-resolution LES is able to predict the drag coefficient fairly well.

[33] For a comparison of the flow fields, horizontal distributions of the time-averaged velocity at half water depth, i.e., $Z / D=5$, of the low-resolution LES (a)-(c)] are presented together with the result from the high-resolution LES (d) in Figure 13. Overall, the distribution of time-averaged streamwise velocity of all grid resolutions are in good agreement with the one predicted by the high-resolution LES. The recirculation zone behind the cylinder is fairly well predicted by the low-resolution LES with the finest grid, but with a decrease in grid resolution, the recirculation length increases. Interestingly, this increase is associated with a decrease in the width of the wake behind the cylinder, which is why the bulk drag coefficients are not negatively affected. This is also reflected in the more quantitative comparison that is provided in Figure 14. The spatially averaged vertical velocity profiles of (a)-(c) are in reasonably good agreement with the high-resolution LES profile regardless of the number of grid cells employed (see Figure 14a). Figure 14b plots the velocity profile along the centerline for one selected cylinder in the flow. The length of the recirculation zone as (a)

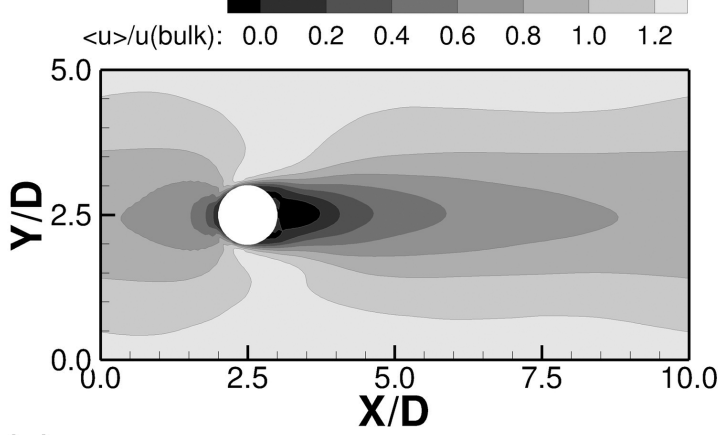

(c)

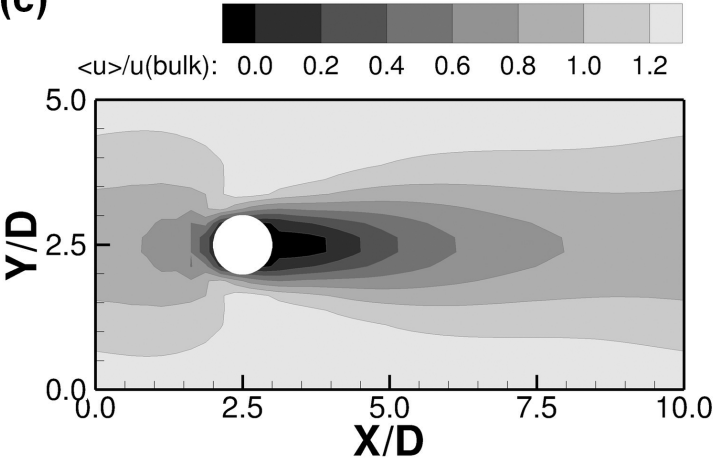

(b)

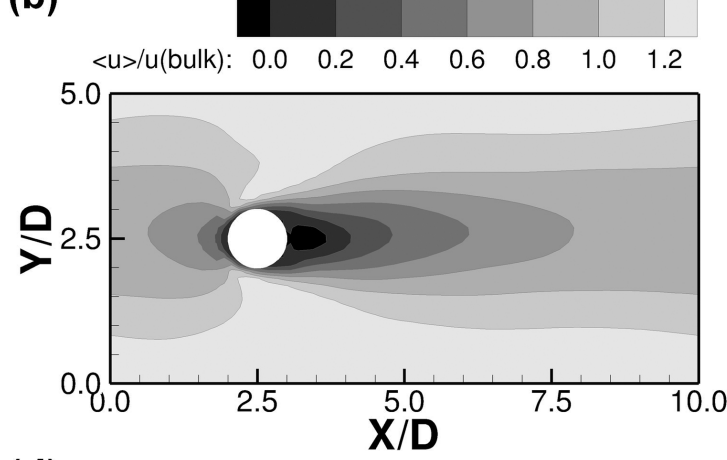

(d)

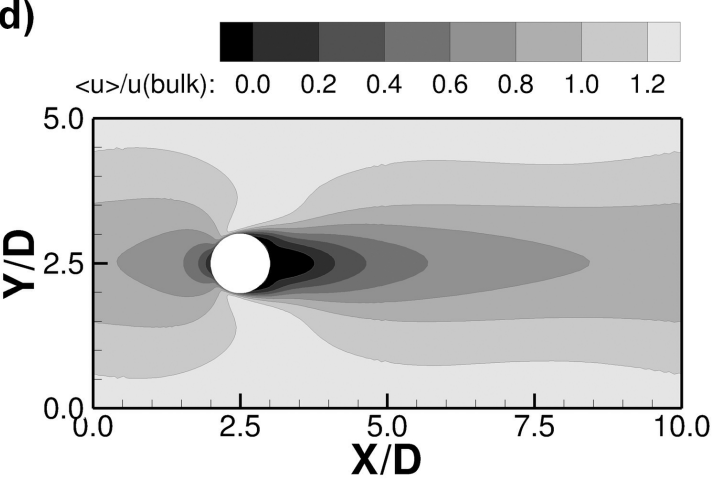

Figure 13. Distribution of time-averaged velocity of the $\phi=0.016$ case at $R e_{D}=500$ simulated on various grids by low-resolution LES, (a)-(c) $(Z / D=5)$, and by (d) high-resolution LES. 
(a)

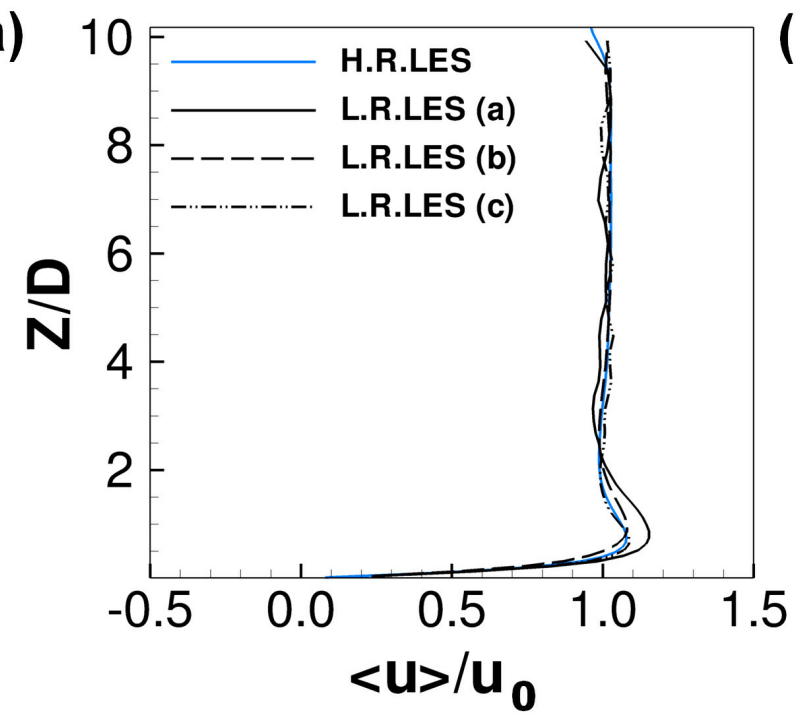

(b)

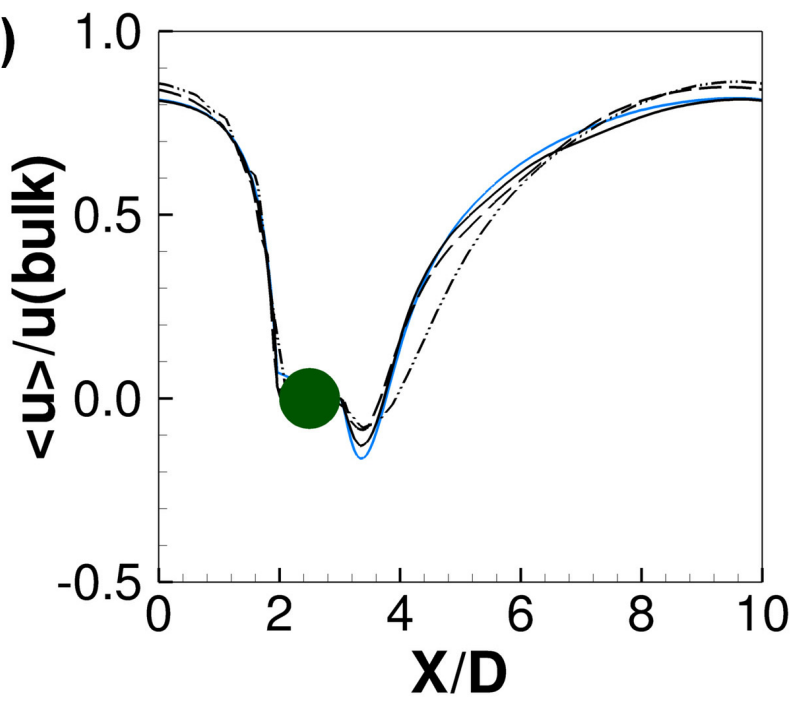

Figure 14. Profiles of (a) spatially averaged streamwise velocity profiles in a vertical direction and (b) time-averaged velocity along centerline for $\phi=0.016$ at $R e_{D}=500$.

well as the velocity gradients are affected by the grid resolution. The coarser the grid, the less resolved the gradients, which results in a mismatch of recirculation zone. Not surprisingly, the best agreement is found with the highest grid resolution; the recirculation zone is overestimated in the simulation on the coarsest grid.

\section{Conclusions}

[34] In this study, the importance of a priori knowledge of drag coefficients in RANS models was investigated first. The RANS simulations performed herein demonstrated that the drag coefficient, an empirical parameter, is key to the accuracy of RANS simulations of flow through vegetation. With a priori knowledge of drag coefficients, accurate flow resistance predictions can be achieved. At high vegetation densities, flow resistance due to vegetation would be severely underestimated if a wrong $C_{D}$ was used. In flow through emergent vegetation, bed friction is assumed negligibly small, and measurements of drag coefficients lump the bed friction into the vegetation drag. When separating bed friction from vegetation drag in RANS simulations of flow through vegetation, predictions of flow resistance in the system are not necessarily improved, at least not for the vegetation densities investigated herein. In order to circumvent the dependency of numerical simulations of flow through vegetation on an empirical parameter, in particular for more complex vegetation arrangements than the ones presented, the method of low-resolution LES is introduced and evaluated. Low-resolution LES has the prospect to compute vegetation drag for complex vegetation arrangements with considerably less computational effort than high-resolution LES and without empirical input. The method combines large-eddy simulations with a first-order immersed boundary method on relatively coarse Cartesian grids. The results indicate that the proposed method provides reasonable accuracy in terms of predicting bulk drag coefficients. It is shown that for low to moderate vegetation densities, velocity gradients, recirculation zones, and secondary flows can be predicted quite reliably. However, uncertainties remain for high vegetation densities.

\section{Notation}

$A_{P} \quad$ projected area of plants

$C_{D}$ drag coefficient

$D$ cylinder diameter

$F_{D} \quad$ drag force

$F_{D \text {,total }}$ total forces exerted on the vegetation

$F_{g}$ total shear stress exerted on the flow region

$g$ acceleration due to gravity $\left(=9.81 \mathrm{~m} \mathrm{~s}^{-2}\right)$

$\stackrel{H}{H}$ water depth

$M$ total number of individual vegetation in flow region

$m$ number of individual vegetation per $\mathrm{m}^{2}$

$R^{2} \quad$ squared correlation coefficient in regression analysis

$R e_{D} \quad$ stem Reynolds number based on cylinder diameter

$S_{0} \quad$ bottom slope of channel

$s$ space between neighboring cylinders

$u_{\text {bulk }}$ fluid velocity in the direction of the mean flow

$u_{\text {cell }}$ calculated velocity by fluid volume fraction

$u_{i} \quad$ initial velocity of each cell

$u_{0}$ free stream velocity

$V_{f}$ fluid volume fraction

Vol total volume of a fluid domain

$\nu$ kinematic viscosity of the working fluid

$\phi \quad$ cylinder volume fraction (= vegetation density)

$\rho$ working fluid density

$\tau_{0} \quad$ global shear stress

$d p / d x \quad$ pressure gradient

[35] Acknowledgments. Su Jin Kim was supported by GA Water Resources Institute 104B grant No. 2009GA197B. Georgia Institute of Technology undergraduate students Ingrid Duque and Emma Reuter helped with collecting laboratory data. We would also like to thank two unknown reviewers for their valuable comments. 


\section{References}

Choi, S. U., and H. Kang (2004), Reynolds stress modeling of vegetated open-channel flows, J. Hydraul. Res., 42(1), 3-11.

Cui, J., and V. S. Neary (2002), Large eddy simulation (LES) of fully developed flow through vegetation, in Hydroinformatics 2002: Proc. Fifth Int. Conf. Hydroinformatics, Cardiff, U. K., pp. 39-44.

Defina, A., and A. C. Bixio (2005), Mean flow and turbulence in vegetated open channel flow, Water Resour. Res., 41, W07006, doi:10.1029/ 2004WR003475.

Dunn, C., F. Lopez, and M. Garcia (1996), Mean flow and turbulence in a laboratory channel with simulated vegetation, Hydraulic engineering Series Rep. 1, Univ. Illinois at Urbana-Champaign.

Ferreira, R. M. L., A. M. Ricardo, and M. J. Franca (2009), Discussion of 'Laboratory investigation of mean drag in a random array of rigid, emergent cylinders' by Yukie Tanino and Heidi M. Nepf, ASCE, J. Hydraul. Eng., 135(8), 690-693.

Fischer-Antze, T., T. Stoesser, P. Bates, and N. R. B. Olsen (2001), 3d numerical modelling of open-channel flow with submerged vegetation, $J$. Hydraul. Res., 39(3), 303-310.

Germano, M., U. Piomelli, P. Moin, and W. H. Cabot (1991), A dynamic subgrid-scale eddy viscosity model, Phys. Fluids, 3(7), 1760-1765.

Ikeda, S., K. Ohta, and H. Hasegawa (1994), Instability-induced horizontal vortices in shallow open-channel flows with an inflection point in skewed velocity profile, JSCE, J. Hydrosci. Hydraul. Eng., 12(2), 69-84.

Kothyari, U. C., K. Hayashi, and H. Hashimoto (2009), Drag coefficient of unsubmerged rigid vegetation stems in open channel flows, J. Hydraul. Res., 47(6), 691-699.

Liu, D., P. Diplas, J. D. Fairbanks, and C. C. Hodges (2008), An experimental study of flow through rigid vegetation, J. Geophys. Res., 113, F04015, doi: $10.1029 / 2008$ JF001042.

Lopez, F., and M. H. Garcia (2001), Mean flow and turbulence structure of open-channel flow through non-emergent vegetation, ASCE, J. Hydraul. Eng., 127(5), 392-402.

Nadaoka, K., and H. Yagi (1998), Shallow-water turbulence modeling and horizontal large-eddy computation of river flow, ASCE, J. Hydraul. Eng., 124(5), 493-500.

Naot, D., I. Nezu, and H. Nakagawa (1996), Hydrodynamic behavior of partly vegetated open channels, ASCE, J. Hydraul. Eng., 122(11), 625-633.

Neary, V. S. (2000), Numerical model for open channel flow with vegetative resistance, in IAHR's 4th Int. Conf. Hydroinformatics, Cedar Rapids, Iowa.

Neary, V. S. (2003), Numerical solution of fully developed flow with vegetative resistance, J. Eng. Mech., 129(5), 558-563.

Nicholas, A. P., and S. J. McLelland (2004), Computational fluid dynamics modelling of threedimensional processes on natural river floodplains, $J$. Hydraul. Res., 42, 131-143.
Patankar, S. V. (1980), Numerical Heat Transfer and Fluid Flow, 214 pp., Taylor and Francis, N. Y.

Rogallo, R. S., and P. Moin (1984), Numerical simulation of turbulent flows, Annu. Rev. Fluid Mech., 16, 99-137, doi:10.1146/annurev.fl.16. 010184.000531.

Schlichting, H. (1962), Boundary Layer Theory, 4th ed., McGraw-Hill, N. Y.

Shimizu, Y., and T. Tsujimoto (1994), Numerical analysis of turbulent open-channel flow over a vegetation layer using a $k-\varepsilon$ turbulence model, JSCE, J. Hydrosci. Hydraul. Eng., 11(2), 57-67.

Smagorinsky, J. (1963), General circulation experiments with the primitive equations, Mon. Weather Rev., 91(3), 99-164.

Stoesser, T. (2010), A physically realistic roughness closure scheme to simulate turbulent channel flow over rough beds within the framework of LES, ASCE, J. Hydraul. Eng., 136(10), 812-819.

Stoesser, T., and V. Nikora (2008), Flow structure over square bars at intermediate submergence: large-eddy simulation study of bar spacing effect, Acta Geophysica, 56(3), 876-893.

Stoesser, T., C. A. M. E. Wilson, P. D. Bates, and A. Dittrich (2003), Application of a $3 \mathrm{~d}$ numerical model to a river with vegetated floodplains, $J$. Hydroinf., 5(2), 99-112.

Stoesser, T., G. Palau-Salvador, W. Rodi, and P. Diplas (2009), Large eddy simulation of turbulent flow through submerged vegetation, Transp. Porous Media, 78, 347-365.

Stoesser, T., S. J. Kim, and P. Diplas (2010), Turbulent flow through idealized emergent vegetation, ASCE, J. Hydraul. Eng., 136(12), 1003-1017.

Stone, H. L. (1968), Iterative solution of implicit approximations of multidimensional partial differential equations, SIAM J. Num. Anal., 5(3), $530-558$.

Tanino, Y., and H. M. Nepf (2008), Laboratory investigation of mean drag in a random array of rigid, emergent cylinders, ASCE, J. Hydraul. Eng., 134(1), 34-41.

Tsujimoto, T., and T. Kitamura (1992), Experimental study on open-channel flow with vegetated zone along sidewall, KHL progressive report, Hydr. Lab., Kanazawa University, Japan in Nadaoka and Yagi (1998) and Xiahoui and $\mathrm{Li}$ (2002), 3, 21-35.

Tsujimoto, T., and Y. Shimizu (1994), Flow and suspended sediment in a compound channel with vegetation, in Proc. 1st Int. Symp. Habitat Hydraulics, pp. 357-369, Trondheim, Norway.

Wu, W., F. D. Shields Jr., S. J. Bennett, and S. S. Y. Wang (2005), A depthaveraged two-dimensional model for flow, sediment transport, and bed topography $n$ curved channels with riparian vegetation, Water Resour. Res., 41, W03015, doi:10.1029/2004WR003730.

S. J. Kim and T. Stoesser, School of Civil and Environmental Engineering, Georgia Institute of Technology, 790 Atlantic Drive, Atlanta, Georgia 30332, USA. (thorsten@ce.gatech.edu) 